\title{
ORIGINAL
}

\section{BROTE DE SHIGELLOSIS EN UN BARRIO DE NIVEL SOCIAL BAJO}

\section{José Luis Chover Lara (1), Antonio Salazar Cifre (1), Miriam Gallego Cruz (2), M. ${ }^{a}$ Luisa Camaró Sala (1), Consuelo Segarra Martínez (3) y M. Carmen Peñalver Blasco (4)}

(1) Secciones de Epidemiología y laboratorio. Centro de Salud Publica de Valencia.

(2) Consultorio del barrio La Coma. Centro de Salud de Paterna.

(3) Servicio de Microbiología. Hospital «Amau de Vilanova».

(4) Servicio de Microbiología. Hospital Universitario «La Fe».

\section{RESUMEN}

Fundamento: Los brotes epidémicos debidos a Shigella sonnei en nuestro entorno se hallan asociados frecuentemente a guarderías y colegios de educación primaria. Se comunica un brote epidémico de shigellosis en un barrio marginal. El objeto del estudio fue identificar la fuente de infección, modo de transmisión, características de los afectados y valorar la pertinencia de las medidas adoptadas.

Métodos: Para el seguimiento temporal del brote, se realizó un estudio observacional longitudinal mixto, en territorio delimitado para las variables clásicas de vigilancia epidemiológica (tiempo, lugar y persona). Se analiza la potencia infectante de la fuente (tasa de ataque) por edad, sexo y centro escolar; razón de tasas y fracción atribuible.

Resultados: Temporalmente el brote se extendió entre las semanas $46 / 97$ y la $8 / 98$. Afectó a 218 personas ( 110 varones y 108 mujeres), que suponen el $5,46 \%$ de la población del barrio. La mayor frecuencia se dio en el grupo de $0-4$ años $(43,6 \%)$, asisten a la guardería el $29,4 \%$ (tasa de ataque de $70,32 \%$ ) con un riesgo relativo de 3,9 (IC 95\%: 2,57-5,93) y fracción atribuible del $74,36 \%$. La razón de tasas entre la guardería y los colegios del barrio es 5,62 (IC 95\%: 4,33-7,31). Se realizó coprocultivo en muestras de 84 casos (38.5\%) aislándose Shigella sonnei en $38(17.4 \%)$. Se pautó tratamiento antibacteriano (amoxi-clavulánico) y se aplicaron medidas higiénico-sanitarias individuales y colectivas.

Conclusiones: El largo período de transmisibilidad y la baja dosis de microorganismos viables necesarios para producir enfermedad, propició una transmisión interpersonal en los ámbitos escolar y familiar. Las medidas aplicadas limitaron de modo eficiente la transmisión del agente infeccioso en los centros escolares.

Palabras clave: Shigellosis. Shigella sonnei. Disentería bacilar. Brote Epidémico.

Correspondencia:

José Luis Chover Lara.

Centro de Salud Pública de Valencia.

C. San Vicente, 83, 1.

46007 Valencia.

Tel. 96.3511112. Fax: 96.3517808.

Correo electrónico: joseluis.chover@sanidad.m400.gva.es.

\section{ABSTRACT \\ Shigellosis Outbreak in a Lower-class District}

Background: The outbreaks of Shigella sonnei in our environment frequently involve day care centers and elementary schools. An outbreak of shigellosis in a lower-class district is reported. The purpose of this study is that of pinpointing the center of infection, the means of contagion, the characteristics of those infected and of assessing the suitability of the measures taken.

Methods: For monitoring the outbreak over time, a combined observational timeline study was conducted within a territory the bounds of which were marked by means of the conventional epidemiological monitoring variables (time, place and individual). The infectivity of the center of infection (contagion rate) is analyzed by age, gender and school; rate ratio and percentage attributable thereto.

Results: On a time-related basis, the outbreak in question started on week $46 / 97$ and ended on week $8 / 98$. This outbreak involved 218 individuals ( 110 males and 108 females) totaling $5.46 \%$ of the district. The highest frequency was found among the $0-4$ age group $(43.6 \%), 29.4 \%$ in day care $(70.32 \%$ contagion rate) with a relative risk of $3.9(95 \% \mathrm{CI}: 2.57-5.93)$ and $74.36 \%$ attributable percentage. The rate ratio between day care and the schools in the district in question is 5.62 (95\% CI: 4.33-7.31). Stool cultures were taken and analyzed in 84 cases (38.5\%), Shigella sonnei being detected in 38 cases $(17.4 \%)$. Antibacterial treatment (amoxilcilin-clavulan) was set out and individual and group health and safety measures were employed.

Conclusions: The long communicability period and the small number of viable microorganisms necessary for causing this disease fostered its being passed on from one person to another at school and in the home. The measures employed effectively confined the contagion of the infectious agent at the schools.

Key words: Shigellosis. Shigella sonnei. Bacterial dysentery. Outbreak. 


\section{INTRODUCCIÓN}

La shigellosis o disentería bacilar (CIE-9: 004) es una infección bacteriana aguda que afecta el intestino grueso y la porción distal del intestino delgado, presenta una distribución mundial y una elevada prevalencia y gravedad en los países en vías de desarrollo. Debido a su cuadro clínico característico (diarrea, fiebre, náuseas, vómitos, cólicos y sangre y moco en las heces en los casos típi$\cos )$, representa una de las diarreas infecciosas diagnosticada y declarada con mayor precisión ${ }^{1}$.

El género Shigella comprende cuatro especies o serogrupos, siendo el más común en los países desarrollados la Shigella son$n e i$, que es el causante de entre el 60 y $80 \%$ de los casos comunicados ${ }^{1-4}$. Otra característica de interés en estos bacilos gramnegativos es el surgimiento de cepas resistentes a múltiples antibióticos, en función del empleo generalizado de agentes antimicrobia$\operatorname{nos}^{2,4,5}$.

La frecuencia máxima de la enfermedad se comunica entre los niños de seis meses a diez años de edad y, a menudo, se presenta en forma de brotes de carácter familiar o comunitario ${ }^{2,6}$. La agregación de casos de disentería bacilar constituye en los países desarrollados un evento epidémico asociado comúnmente a deficientes condiciones higiénicas, en grupos calificados tradicionalmente de alto riesgo por causa social ${ }^{7}$.

Los brotes epidémicos debidos a Shigella sonnei en el entorno europeo se hallan asociados, frecuentemente, a guarderías y colegios de educación primaria-10. El modo habitual de transmisión es el contacto «persona a persona», siendo el vehículo de contagio las manos de los sujetos afectados y/o de sus cuidadores'. Con independencia de otros vehículos descritos como lechuga ${ }^{11,12}$, agua $^{13,14}$, cubitos de hielo ${ }^{13}$ o maí ${ }^{15}$, la transmisión interpersonal en centros escolares o en familias con escasos recursos económicos y culturales aporta el mayor volu- men de casos y las principales situaciones de carácter epidémico.

El estudio que se presenta corresponde a un brote epidémico de shigellosis de ámbito comunitario ocurrido en el barrio La Coma de Paterna (Valencia). La sospecha del brote fue comunicada en la semana 49 de 1997 por el equipo de Atención Primaria tras la confirmación del laboratorio de los casos de diarrea que venían observando durante las semanas anteriores.

El objeto del estudio fue identificar la fuente de infección y modo de transmisión, así como las características de los afectados y valorar la pertinencia de las medidas adoptadas.

La Coma constituye un barrio completamente aislado del núcleo urbano del municipio al que pertenece, compuesto por cerca de 1.200 viviendas, todas ellas de promoción pública, destinadas a albergar familias con escasos recursos económicos, incapacitadas para acceder a la vivienda en el mercado libre inmobiliario. Declarado por la Generalitat Valenciana como «barrio de acción preferente» (Decreto 157/1988 de 11 de octubre. Plan conjunto de actuaciones en Barrios de Acción Preferente) presenta las características propias de un barrio marginal: ausencia de empresas y comercio, alta tasa de desempleo - particularmente agudo entre los jóvenes -, absentismo y fracaso escolar alto, falta de cualificación profesional, elevada mortalidad en jóvenes, relacionada con el consumo drogas y la infección por VIH etc. (Comisión gestora. Proyecto de Plan Integral de Desarrollo Local del Barrio de la Coma. Paterna: Comisión Gestora; 1988). De acuerdo con el censo de 1996, el barrio cuenta con un total de 3.991 habitantes, con la distribución por edad y sexo recogida en la pirámide poblacional (figura 1).

\section{MATERIAL Y MÉTODOS}

Para el seguimiento temporal del brote, se realizó un estudio observacional longitudi- 
Figura 1

Pirámide de edad del harrio La Coma y distribución de casos por grupos de edad y sexo

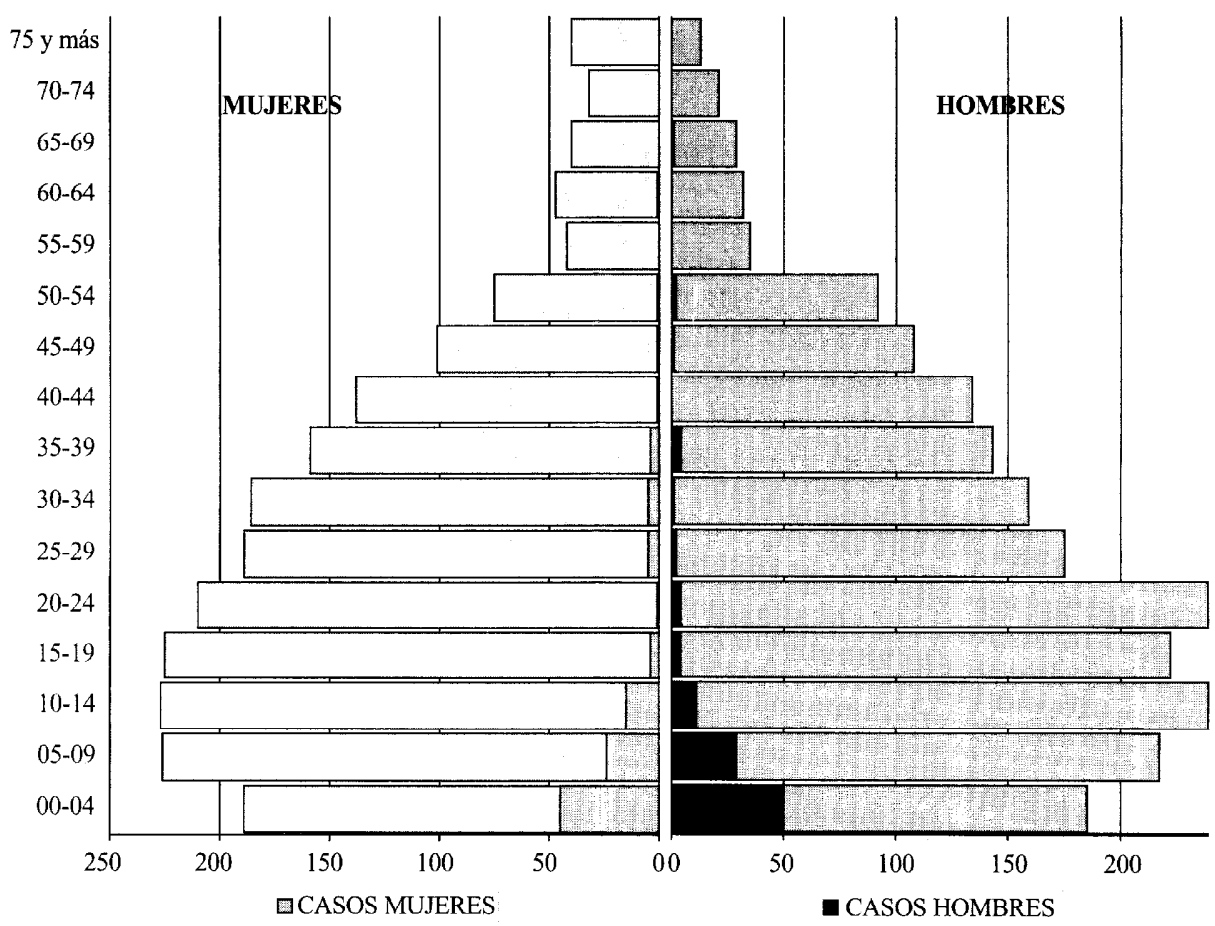

nal mixto (restrospectivo-prospectivo) en territorio delimitado para las variables clásicas de vigilancia epidemiológica (tiempo, lugar y persona) valorando la distribución espacial, frecuencias por grupos de edad y scxo, frecuencia de síntomas y tasas de ataque por edad y centro escolar.

Ante la notificación de los casos iniciales, se procedió a la recogida de datos con la incorporación de los casos observados en las semanas anteriores, utilizando el documento de encuesta de disentería bacilar de la Red Nacional de Vigilancia Epidemiológica ${ }^{17}$, con el fin de clasificar los casos y los factores asociados a la transmisión. Se procedió también a la revisión de los registros microbiológicos de los hospitales del Área de Salud para establecer la secuencia temporal y la agregación geográfica.
Esta recuperación de registros permitió la siguiente clasificación de casos: caso confirmado cuando se ha dado un aislamiento de Shigella de una muestra clínica y caso probable en los clínicamente compatibles y epidemiológicamente relacionados con un caso confirmado ${ }^{16,17}$.

El estudio microbiológico de heces (coprocultivos) se realizó en los laboratorios de Microbiología de los hospitales $\mathrm{La} \mathrm{Fe} \mathrm{y}$ Arnau de Vilanova y en el del Centro de Salud Pública de Valencia, siguiendo el método microbiológico tradicional y el protocolo existente para este tipo de muestras. Para el aislamiento de las cepas se utilizaron los medios comerciales Agar SS y McConkey. Las cepas aisladas fueron serotipificadas mediante técnicas de aglutinación utilizando medios específicos frente a Shigella son- 
nei, $S$. boydii, $S$. dysenteriae y $S$. flexneri y finalmente, se estudió el perfil de sensibilidad a antimicrobianos, en placas conteniendo Agar Müeller-Hinton. Los antibióticos ensayados fueron: amoxicilina/clavulánico, norfloxacina, ácido nadilíxico, ácido pipemídico, ciprofloxacina, ampicilina y cotrimoxazol.

La sección de Calidad Ambiental de la Dirección Territorial de Medio Ambiente realizó la inspección de la red de suministro de agua de la instalación y tomó muestras de agua de diversos puntos de la red y de las fuentes conectadas, las cuales fueron analizadas en sus caracteres microbiológicos, organolépticos y físico-químicos.

Se analizó la potencia infectante de la fuente (tasa de ataque) por edad y centro escolar; razón de tasas y fracción atribuible (expuestos) aplicando la fórmula $F a_{e}=I_{e}-I_{n e} / I_{e}$ donde $I_{e}$ es la tasa de incidencia entre los expuestos e $I_{n e}$ la tasa de inci- dencia entre los no expuestos. Se estableció la asociación e identificación del origen mediante los criterios mayores de causalidad: fuerza de la asociación, temporalidad, plausibilidad biológica y cesación del fenómeno. Se realizó un análisis estadístico de frecuencias y se compararon las variables cualitativas (sexo, centro escolar), estimándose el riesgo relativo ( $R R$ ), su intervalo de confianza de $95 \%\left(\mathrm{IC}_{95 \%}\right)$ y significación mediante la prueba de chi-cuadrado $\left(\chi^{2}\right)$. La creación y gestión de la base de datos se realizó con el programa DBASE III y los análisis estadísticos de los resultados se efectuaron con el programa SPSS para Windows versión 6.1.3.

\section{RESULTADOS}

El brote debutó el 10/11/97 con 4 casos en la Guardería La Coma donde se mantuvo casi exclusivamente hasta la semana 48 (figura 2). Franqueada la barrera de la guar-

Figura 2

Brote de shigellosis: curva epidémica y distribución de los casos según procedencia

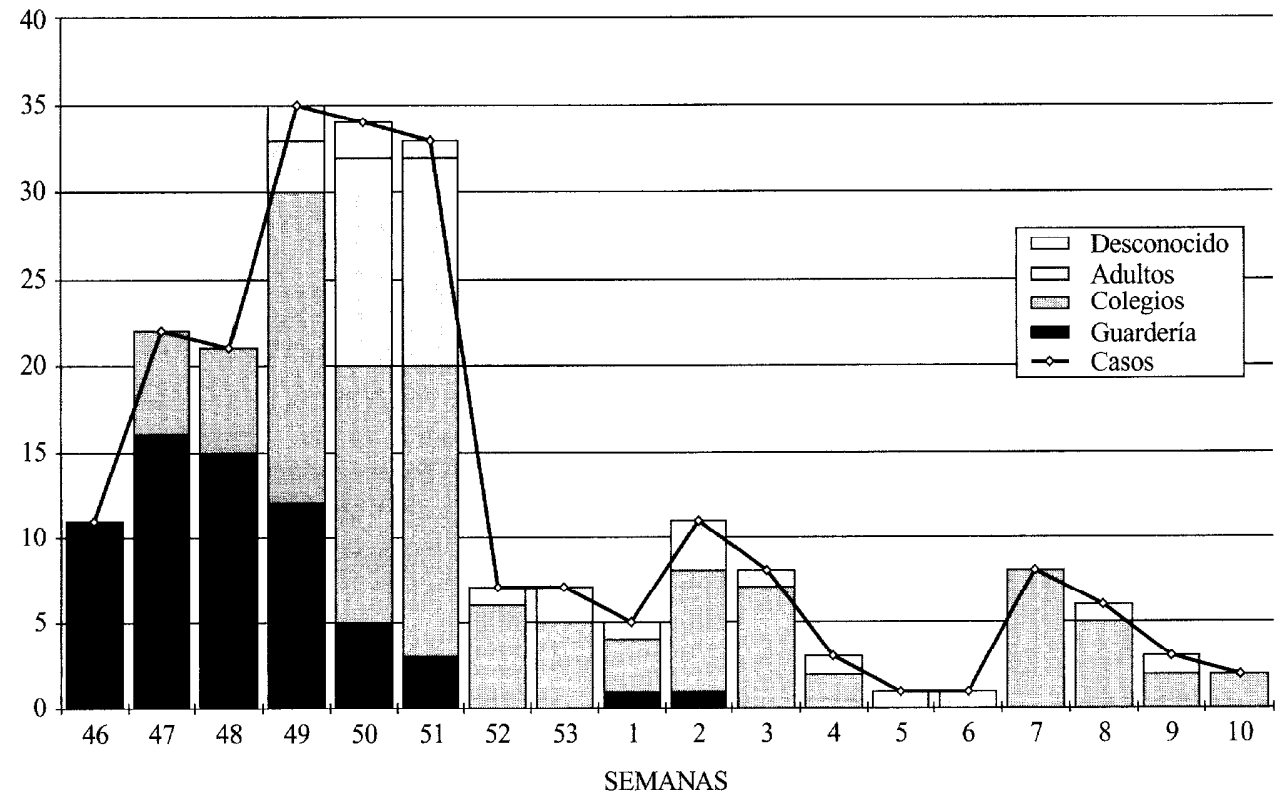


dería, se observa una curva creciente, situándose el pico en las semanas 49,50 y 51 con 35,34 y 33 casos respectivamente. Temporalmente se extendió entre las semanas 46 del 97 y la 8 del 98, registrándose algunos casos aislados en la cola del brote, los cuales se han incorporado. La curva presenta como características su prolongación en el tiempo y un aspecto plurimodal que se expresa mediante diversas ondas o generaciones de casos sucesivas (figura 2). En la figura 3 se representan los casos confirmados y probables por semana de inicio de síntomas.

Figura 3

Brote de shigellosis: casos confirmados y probables por semanas de presentación

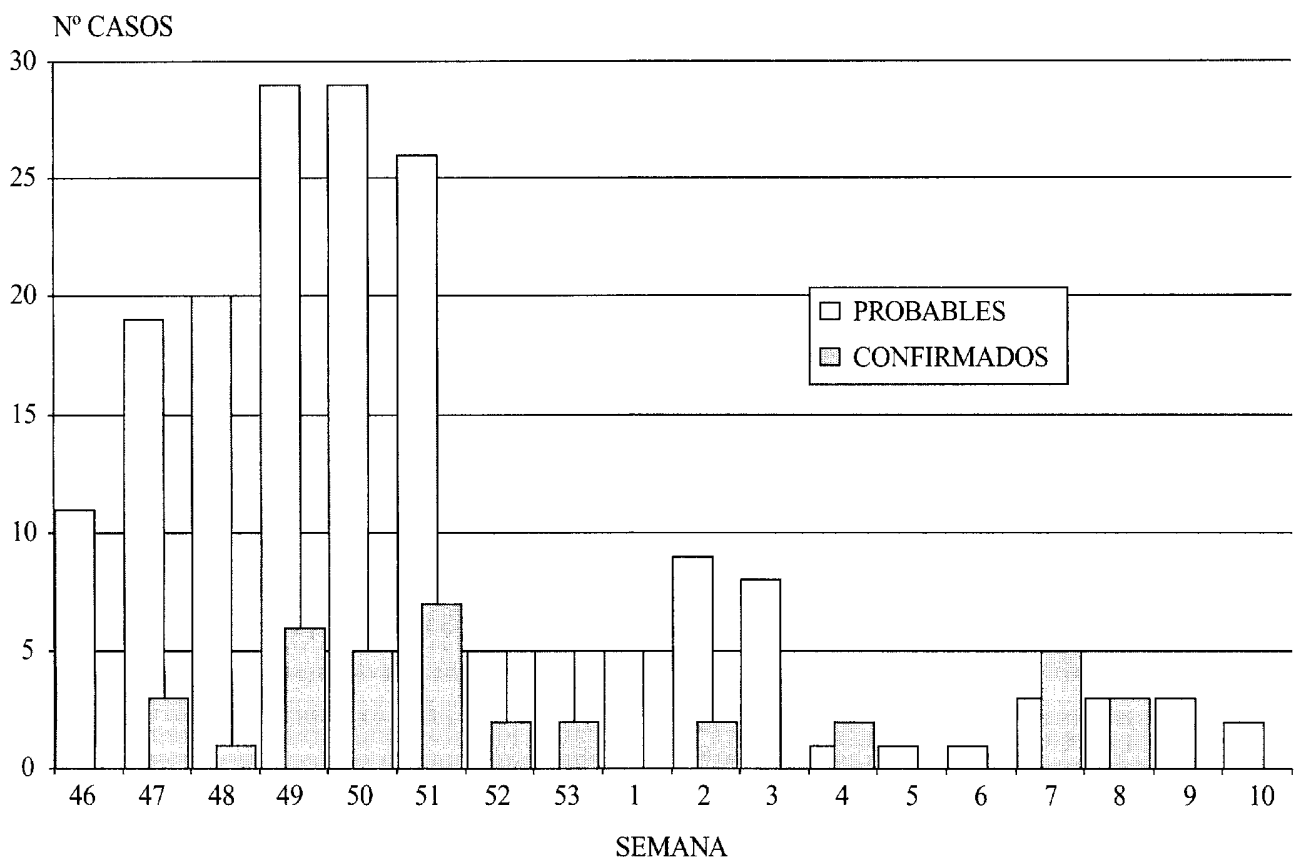

El brote afectó a 218 personas, de las cuales $110(50,45 \%)$ son varones y 108 $(49,54 \%)$ mujeres; sin diferencias de riesgo por sexo. El total de afectados suponen el $5,46 \%$ de la población del barrio, y 11 de los casos precisaron hospitalización.

La mayor frecuencia por edad se dió en el grupo de 0-4 años con el 43,6\% de los casos, seguido del grupo 5-9 con el 24,3 (figura 1). Los menores de 2 años alcanzan el 14,7\%, mientras que el $67,9 \%$ de los casos tiene menos de 10 años y $79,8 \%$ menos de 15 .
Considerado globalmente el barrio, enfermaron el $34,05 \%$ de todos los sujetos pertenecientes al grupo de edad más afectado. La mediana sc situó cn los 5 años.

El número y porcentaje de casos que asistían a los distintos centros escolares, las tasas de ataque y el RR estimado sobre la población censada en el barrio de los correspondientes grupos de edad se presentan en la tabla 1. La razón de tasas de ataque entre la guardería y la suma de los dos colegios del barrio fué de 5,62 (IC $95 \%$ : 
4,33-7,31; $\mathrm{p}<0,0001)$. Considerada la asistencia a la guardería como factor de exposición ambiental para el grupo de edad de 0-3 años, se observó que de los 91 niños que asisten a la guardería enfermaron 64 (TA 70,32\%) mientras que de los 111 restantes que completan el censo del barrio en este tramo de edad y que no asisten a la guardería, enfermaron solamente 20 (TA 18,0\%). El RR se sitúa en 3,9 $\left(\mathrm{IC}_{95 \%}: 2,57-5,93\right)\left(\chi^{2} 56,05 \mathrm{p}<0,0001\right)$ y la fracción atribuible para la asistencia a la guardería en este grupo de edad fue del $74,36 \%$.

Tabla 1

Distribución de los casos según colegio al que asisten, tasa de ataque y riesgo relativo (RR)

\begin{tabular}{|c|c|c|c|c|c|c|c|}
\hline \multirow{2}{*}{ Colegio } & \multicolumn{2}{|c|}{ Alumnos } & \multicolumn{2}{|c|}{ Enfermos } & \multirow{2}{*}{$\begin{array}{c}\text { Tasa de ata- } \\
\text { que (\%) }\end{array}$} & \multirow{2}{*}{$R R^{*}(I C 95 \%)$} & \multirow{2}{*}{$p$} \\
\hline & Grupo Edad & $n$ & $n$ & $\%$ & & & \\
\hline Guardería La Coma & $0-3$ a. & 91 & 64 & 29,4 & 70,32 & $3,9(2,57-5,93)$ & 0,00000 \\
\hline$<4$ años que no asisten a guardería & $0-3 \mathrm{a}$. & 111 & 20 & 9,2 & 18,0 & & \\
\hline \multirow[t]{4}{*}{ Colegio P. La Coma } & $4 \mathrm{a}$. & 31 & 3 & & 9,68 & & \\
\hline & $5-9$ a. & 163 & 30 & & 18,40 & & \\
\hline & $10-14$ a. & 136 & 12 & & 8,82 & & \\
\hline & Totales & 330 & 45 & 20,6 & 13,51 & $1,75(1,18-2,59)$ & 0,004 \\
\hline \multirow[t]{4}{*}{ Colegio P. Antonio Ferrandis } & 4 a. & 34 & 6 & & 17,65 & & \\
\hline & $5-9$ a. & 89 & 12 & & 13,48 & & \\
\hline & $10-14$ a. & 72 & 3 & & 4,16 & & \\
\hline & Totales & 195 & 21 & 9,9 & 10,76 & $1,11(0,70-1,77)$ & 0,64 \\
\hline Otros colegios/institutos ESO & & & 23 & 10,5 & & & \\
\hline Adultos & & & 40 & 18,3 & & & \\
\hline Desconocido & & & 5 & 2,3 & & & \\
\hline TOTAL & & & 218 & 100 & & & \\
\hline
\end{tabular}

* El RR se establece en relación a la población del barrio de los grupos de edad correspondientes.

La relación escolar entre los casos se manifiesta en el $60,6 \%$ y la relación familiar en el $67,4 \%$. En el $39,4 \%$ del total se observaron casos en su entorno escolar y familiar y en $25(11,46 \%)$ se desconoce relación.

La diarrea, presente en el $81,2 \%$ de los casos, fue el síntoma más frecuente, seguido de fiebre $(56,9 \%)$, dolor abdominal $(52,3 \%)$ $\mathrm{y}$ vómitos $(47,7 \%)$, detectando sangre/moco en heces en el $22,0 \%$. Se realizó coprocultivo en muestras de 84 casos $(38,5 \%)$ y de 8 no casos/manipuladores de alimentos de los centros escolares. Se aisló Shigella sonnei en 38 , que confirma el diagnóstico en el
$17,4 \%$ de los casos. La cepa aislada en todos ellos presentó un perfil de sensibilidad antimicrobiana idéntico; resultó sensible a los antibióticos amoxicilina/clavulánico y norfloxacina, y resistente a ampicilina y cotrimoxazol. En los no casos/manipuladores de alimentos el resultado de los coprocultivos fue negativo y los análisis microbiológicos practicados en el agua de consumo público que suministra al barrio no detectaron la presencia de patógenos.

Se prescribió aislamiento (no asistencia al colegio) y pautó un tratamiento antibacteriano (amoxicilina-clavulánico) a todos los ca- 
sos de diarrea que se fueran presentando, con el fin de reducir la diseminación secundaria de la infección, acortando el curso e intensidad de la enfermedad y la duración de la excreción del agente patógeno.

Al mismo tiempo, se establecieron medidas higienico-sanitarias individuales y colectivas durante la fase aguda de la enfermedad con el lavado eficaz, frecuente y supervisado de las manos con agua corriente con hipoclorito sódico al $2 \%$ en los colegios y guardería. El brote remitió en la semana 52, tres después del inicio de la intervención.

\section{DISCUSIÓN}

La disentería bacilar es la más transmisible de las diarreas bacterianas. Experimentos realizados han demostrado que la shigellosis es única entre las enfermedades causadas por enteropatógenos bacterianos, en el sentido de que menos de 200 células viables producen fácilmente la enfermedad ${ }^{2.18}$. Esta baja dosis de microorganismos necesarios explica la frecuencia de la transmisión interpersonal y que la disentería sea un problema importante en poblaciones hacinadas o en centros institucionales ${ }^{2,6}$. El control de un brote comunitario de shigellosis puede presentar cierta dificultad por la facilidad de la transmisión entre niños pequeños, por su alta tasa de ataque secundario, por la frecuentemente larga duración de los brotes y por los múltiples puntos de exposición ${ }^{6}$. Un requisito casi necesario en la transmisión de la enfermedad es el grado de contacto y el nivel de higiene personal entre los pacientes afectos y las personas susceptibles.

En el presente brote, las características de la curva epidémica se corresponden con una exposición masiva común seguida de casos secundarios, mecanismo que ocurre cuando se trata de una enfermedad muy difusible y/o cuando las condiciones ambientales de hacinamiento y otras son tales que favorecen la propagación de la enfermedad ${ }^{20}$. El período de transmisibilidad prolongado (hasta 4 semanas sin tratamiento antimicrobiano) y la baja dosis de microorganismos viables necesarios propiciaron la transmisión interpersonal en los ámbitos escolar y familiar. El modo de transmisión también se confirma por el curso del brote, su larga duración y la exclusión de otras fuentes de contagio como el agua potable y los alimentos.

Las relaciones escolares fueron determinantes en el inicio y desarrollo del brote, no habiéndose podido establecer un caso índice, por faltar el diagnóstico de algún caso que haya actuado de nexo entre los primeros del brote y varios casos aislados que había comunicado el servicio de Microbiología del hospital La Fe en los meses de septiembre y octubre en niños residentes en el barrio.

La intensidad de la asociación pone de manifiesto el papel de la guardería como foco inicial, evidencia que viene corroborada por la secuencia temporal en la aparición de los casos secundarios por contagio entre los hermanos. Durante las semanas 46, 47 y 48 , los casos se mantuvieron circunscritos a la guardería La Coma, donde se ha producido la tasa de ataque más alta, siendo tratadas las diarreas, en aquel momento, con dieta y reposición de líquidos. En estos centros, la transmisión cstá favorccida por las prácticas habituales de las cuidadoras tales como cambio de pañales y/o asistir a los niños de 2-3 años en el aseo, acciones que suelen simultanear con la manipulación de alimentos al dar de comer a los niños ${ }^{21}$, aspectos que no han podido evidenciarse en el presente brote, aunque tampoco se observó la aplicación de medidas de higiene personal estrictas, siendo estas prácticas momentos claros de riesgo ${ }^{10}$.

A partir de la semana $47-48$ se produjo una segunda onda epidémica, debida a la transmisión intrafamiliar, que afecta fundamentalmente a los hermanos de los niños de la guardería, apareciendo los primeros casos secundarios en los otros colcgios del barrio, 
lo cual facilitó el mantenimicnto y desarrollo del brote al asegurar la presencia de nuevos susceptibles. Esta transmisión familiar también permitió la afectación de adultos y niños/lactantes que no asisten a la guardería o a los centros escolares.

La distribución de los casos por grupos de edad observada es coincidente con lo descrito en la literatura, ya que la disentería bacilar es una enfermedad que afecta principalmente a los niños entre los 6 meses y los 10 años de edad, alcanzando las dos terceras partes de los casos comunicados ${ }^{2,6}$. Llama la atención que en los adultos afectados, el $76 \%$ de los comprendidos entre 25 y 34 años son mujeres, lo cual se explicaría por el contacto más frecuente y prolongado de las madres con sus hijos de corta edad.

La administración extensiva de profilaxis antibacteriana procede, según nuestro criterio, cuando las circunstancias de crecimiento de casos y reinfección de los mismos es uniformemente acelerado, el aislamiento geográfico y físico de la población están presentes y las condiciones sociales y culturales pueden agravar o extender en el tiempo una patología que normalmente es banal o levementc agresiva. Corrobora csta apreciación, los antecedentes conocidos en la literatura científica y, fundamentalmente, la cesación del fenómeno observada al pautar profilaxis extensiva, la cual ha ejercido una acción básica en el control del brote al detener el crecimiento del número de sujetos portadores y reducir el período de evacuación de gérmenes ${ }^{22}$. Por su parte, las medidas higienico-sanitarias establecidas - aplicadas y recomendadas en numerosos trabajos - contribuyeron a limitar de un modo eficiente la transmisión en los centros escolares $^{20-23}$.

Las características sociales y de marginalidad del barrio La Coma, en el que se dan situaciones de hacinamicnto dc familias $y$ de hábitos de higiene personal deficientes, pueden haber influido negativamente en el desarrollo, mantenimiento y duración del brote, mientras que el retraso diagnóstico de los primeros casos de shigellosis y la no sospecha inicial de la situación de brote fue determinante, en gran medida, de la dimensión del mismo.

\section{BIBLIOGRAFÍA}

1. Dupont, Herbert L. Especies de Shigella (Disentería bacilar) En: Mandell, Douglas y Bennett. Enfermedades Infecciosas. Principios y práctica, 4. ${ }^{a}$ edición. Buenos Aires: Panamericana; 1997. p. $2276-83$.

2. Benenson, Abram S. (Editor) Manual para el control de las enfermedades transmisibles. 16. ${ }^{\mathrm{a}}$ edición. OPS Publicación científica n. ${ }^{\circ}$ 564; 1997. p. $412-6$.

3. Castillo FJ; Carranza E; Clavel A; Rubio MC; Gomez-Lus R. Epidemiología de shigellosis y colicinotipia de Shigella sonnei. Un estudio de 14 años. Enferm Infecc Microbiol Clin 1991; 9: 530-6.

4. Yurdak"ok K; Sahin N; Ozmert E; Berkman E. Shigella gastroenteritis: clinical and epidemiological aspects and antibiotic susceptibility. Acta Paediatr Jpn 1997; 39: 681-4.

5. Wharton M; Spiegel RA; Horan JM; Tauxe RV; Wells JG; Barg N; Herndon J; Meriwether RA; MacCormack JN; Levine RH. A large outbreak of antibiotic-resistant shigellosis at a mass gathering. J Infect Dis 1990, 162: 1324-8.

6. Current trends community outbreaks of shigellosis - United States. MMWR 1990; 39: 509-13, 519.

7. Lee LA; Shapiro CN; Hargrett-Bean N; Tauxe RV. Hyperendemic shigellosis in the United States: a review of surveillance data for 1967-1988. J Infect Dis 1991; 164: 894-900.

8. Evans HS; Maguire H. Outbreaks of infectious intestinal disease in schools and nurseries in England and Wales 1992 to 1994. Commun Dis Rep CDR Rev 1996; 6: R103-8.

9. Caldwell GG; Fiegel D; Bryant L; Chambers D; Rask P. Shigella in Tulsa County, 1993: epidemiology, day care center association, and control. J Okla State Med Assoc, 1995; 88: 198-204.

10. Shigellosis in child day care centers - Lexinton- Fayette County, K. MMWR 1992; 41: 440-2. 
11. Frost JA; McEvoy MB; Bentley CA; Anderson Y; Rowe B. An outbreak of Shigella sonnei Infection associated with consumption of iceberg lettuce. Emerg Infect Dis 1995; 1: 26-9.

12. Kapperud G; Rorvik LM; Hasseltvedt V; Hoiby EA; Iversen BG; Staveland K; Johnsen G; Leitao J; Herikstad H; Andersson Y; et al. Outbreak of Shigella sonnei infection traced to imported lettuce. J Clin Microbiol 1995, 33: 609-14.

13. Shigella sonnei outbreak associated with contaminated drinking water - Island Park, Idaho, August 1995. MMWR 1996; 45: 229-231.

14. Morera MA; Espejo E; Coll P; Simó M; Uriz MS; Llovet T; Rodríguez M; Martinez A; March F; Bella F. Brote epidémico de shigellosis asociado al consumo de agua. Enferm Infecc Microbiol Clin 1995; 13: 160-5.

15. Molbak K; Neimann J. Outbreak in Denmark of Shigella sonnei infections related to uncooked «baby maize» imported from Thailand. Eurosurveillance Weekly 1998; 2: 980813.

16. Centro Nacional de Epidemiología. Protocolos de las Enfermedades de Declaración Obligatoria. Madrid: Ministerio de Sanidad y Consumo; 1996.
17. CDC. Case definitions for Infectious Conditions Under Public Health Surveillance. MMWR 1997; 46 (n. ${ }^{\circ}$ RR-10): 31-32.

18. Crockett CS; Haas CN; Fazil A; Rose JB; Gerba CP. Prevalence of shigellosis in the U.S.: consistency with dose-response information. Int J Food Microbiol 1996; 30: 87-99.

19. Armijo R. Epidemiología básica en atención primaria de salud. Madrid: Díaz de Santos; 1994.

20. Mohle-Boetani JC; Stapleton M; Finger R; Bean $\mathrm{NH}$; Poundstone J; Blake PA; Griffin PM. Communitywide shigellosis: control of an outbreak and risk factors in child day-care centers. Am J Public Health 1995; 85 (6): 812-6.

21. Multistate outbreak of Shigella sonnei gastroenteritis - United States. MMWR 1987; 36: 440-2, 448-9.

22. Hoffman RE; Shillam PJ. The usc of hygicne, cohorting, and antimicrobial therapy to control an outbreak of shigellosis. Am J Child 1990; 144: 219-221.

23. Mahoney FJ; Farley TA; Moriniere BJ; Winsor DK; Silberman RL; McFarland LM. Evaluation of an intervention program in the control of an urban outbreak of shigellosis. Am J Prev Med 1991; 7: 292-7. 